\title{
Repeated Live Imaging in the Temporomandibular Joint of an Anterior Crossbite Mouse Model Using a 7T Magnetic Resonance Device
}

\author{
KO ITO ${ }^{1}$, FUMIE YAMAZAKI ${ }^{1,2}$, SHINNOSUKE NOGAMI ${ }^{3}$, KOSUKE TAKAHASHI $^{1}$, \\ TOSHIROU KONDOH ${ }^{1}$ and ALASTAIR GOSS ${ }^{4}$ \\ ${ }^{1}$ Department of Maxillofacial Surgery, Nihon University School of Dentistry at Matsudo, Matsudo, Japan; \\ ${ }^{2}$ Department of Regulatory Physiology, Dokkyo Medical University School of Medicine, Mibu, Japan; \\ ${ }^{3}$ Division of Oral and Maxillofacial Surgery, Department of Oral Medicine and Surgery, \\ Tohoku University Graduate School of Dentistry, Sendai, Japan; \\ ${ }^{4}$ Oral and Maxillofacial Surgery Unit, Faculty of Health Science University of Adelaide, Adelaide, Australia
}

\begin{abstract}
Background/Aim: Few studies have performed magnetic resonance $(M R)$ imaging on live animals. The aim of this study was to perform $7 T$ MR microimaging of the temporomandibular joint (TMJ) multiple times in the same living mice with malocclusion, and to compare between MR imaging and histopathological findings. Materials and Methods: Mice were examined by MR imaging at 3-4, 6 and 12 weeks following the attachment of a metal tube on the left mandibular incisor. Histopathological examination was done at 3, 6 and 12 weeks. Results: The detailed structure of the TMJ was evident from MR microimaging. The histopathological examination showed some changes in the cartilage, but no changes in the bone structure of these mice. Conclusion: We successfully performed multiple 7T MR imaging in living mice. Even if the TMJ showed no obvious changes on MR images, minute changes may be present in the cartilage.
\end{abstract}

Internal derangement (ID) and osteoarthritis (OA) are relatively common diseases affecting the temporomandibular joint (TMJ). ID is a functional disorder of the articular disc, whereas $\mathrm{OA}$ is considered as a degenerative process, in which cartilage destruction of the mandibular condylar and articular joint surface is accompanied by bone resorption or

This article is freely accessible online.

Correspondence to: Ko Ito, Department of Maxillofacial Surgery, Nihon University School of Dentistry at Matsudo, 2-870-1 Sakaecho-Nishi, Matsudo, Chiba, 271-8587, Japan. Tel: +81 473609395, Fax: +81 473609398, e-mail: ito.ko@nihon-u.ac.jp

Key Words: MR microimaging, live imaging, cartilage, malocclusion, temporomandibular joint. addition (1-3). Histopathological examination of these conditions in humans is limited. Computed tomography (CT) and magnetic resonance (MR) imaging are used in clinical practice and are often applied to determine the treatment strategies and evaluate their effects. There are only few studies which have radiologically evaluated the results of histopathological findings using animal disease models (4, $5)$. Thus, the relationship between actual pathological changes and radiological findings therefore remains unclear.

In animal experiments, MR imaging of animals following sacrifice does not completely reflect the blood flow and interstitial fluid in the living body, so an in vivo imaging acquisition is more accurate. In addition, observing temporal changes in the same animal model is important. This is because pathological changes occurring in the TMJ could change over time $(6,7)$. The ability to carry out examination of this anatomical location in the same experimental animal several times would therefore be highly valuable in terms of elucidating the etiology of joint disease. Although experimental research using large animals, such as sheep and pigs that have similar structures and movement compared to the human TMJ is desirable, equivalent large-scale experimental facilities and imaging devices for large animals are also required, placing practical limitations on the experiments that can be conducted (8-10). Experimental studies using rats and mice are, thus, considered, however, MR imaging of such small animals is not technically easy, mainly due to size limitations and fragility of bone structures. Despite these difficulties, Yamazaki et al., have successfully examined the mouse TMJ using a 7T high-field MR imaging system (5).

In this study, we performed MR imaging several times in the same mice in vivo, following an occlusal disorder that was created by attaching a metal tube to a mandibular 

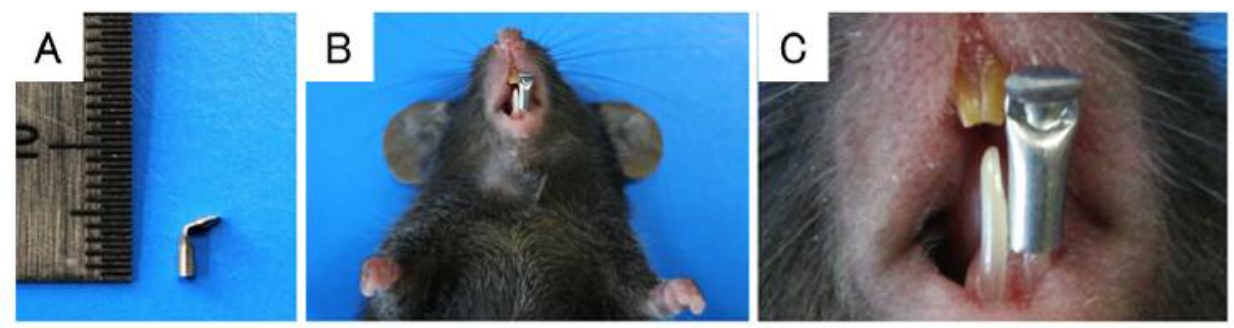

Figure 1. Anterior crossbite mouse model. (A) Metal crowns extending to a height of $5 \mathrm{~mm}$ were made. (B, C) The metal crown was fixed to the mandibular incisor using zinc phosphate cement.

anterior tooth, for the purpose of evaluating and comparing histopathological and MR imaging findings.

\section{Materials and Methods}

The Animal Research Councils of Dokkyo Medical University approved all animal experiments. The related judgment reference number at Dokkyo Medical University is 0876.

Preparation of anterior crossbite for mice. In this study, 8 male C57BL6 mice (age range=6-8 weeks) were divided into two experimental groups: one group was examined by MR imaging prior to the attachment of the metal tube, and then at 3 and 4 weeks after the experimental intervention; while the other group was examined prior to the attachment of the metal tube, and then at 6 and 12 weeks following the experimental operation $(n=4$ each). In each experimental group, a metal tube was bonded onto the left mandibular incisor to create a unilateral anterior crossbite relationship. As described in a previous study (11), the metal tube was made from a pinhead (Shinva Ande, Shandong, PR China; inner diameter $=0.94 \mathrm{~mm}$, thickness $=0.26 \mathrm{~mm}$ ). The mandibular tube was cut to a length of $5 \mathrm{~mm}$, and was bent labially $3 \mathrm{~mm}$ above the anatomical cervical line of the mandibular incisor. The tube was carefully bonded with zinc phosphate cement (GC Co., Tokyo, Japan) under anesthesia using intraperitoneal injection of $50 \mathrm{mg} / \mathrm{kg}$ pentobarbital and was checked every other day. No prostheses fell off during the experimental period. Results of MR imaging prior to the attachment of the metal tube were used as control data. Figure 1 shows the occlusal relationship of the anterior teeth immediately following bonding of the metal tube. Body weight was recorded every other day.

MR microimaging of the mouse TMJ. The metal tube was removed under intraperitoneal anesthesia before MR imaging. After removal, the mouse was set on the 7T MR imaging system, then an 18-mm-diameter surface coil was set on the top of the head to image both right and left TMJs simultaneously. Mice were anesthetized using $1 \%$ sevoflurane in a gas mixture of $\mathrm{O}_{2} / \mathrm{CO}_{2} / \mathrm{N}_{2} \mathrm{O}$ delivered through a face mask at a rate of $0.6 \mathrm{l} / \mathrm{min}$. MR images were obtained using the $7 \mathrm{~T}$ microimaging system (AVANCE III; Bruker BioSpin, Ettlingen, Germany) with a ParaVision operating software (version 5.1; Bruker BioSpin). Parameters for 2-dimensional (2D) $\mathrm{T}_{1}$-weighted $\left(\mathrm{T}_{1} \mathrm{~W}\right)$ gradient echo imaging were: i) field of view (FOV) of $16.64 \times 16.64 \mathrm{~mm}$, ii) matrix of $256 \times 256$, iii) slice thickness of $0.5 \mathrm{~mm}$, iv) repetition time (TR) of $150 \mathrm{~ms}, \mathrm{v}$ ) echo time (TE) of $4 \mathrm{~ms}$, and vi) flip angle at $45^{\circ}$. Fat-suppressed 3-dimensional (3D) T1W was also conducted with i) a voxel size of $80 \times 80 \times 80 \mu \mathrm{m}$, ii) TR at $100 \mathrm{~ms}$, iii) TE at $4 \mathrm{~ms}$, and iv) flip angle at $90^{\circ}$. In accordance with the slice settings for the mouse TMJ (5), we defined the coronal and sagittal planes for MR imaging.

Histological examination. Murine TMJs were dissected after MR imaging at 3,6 , or 12 weeks following tube bonding $(n=2$ at each time point), were then fixed in $10 \%$ neutral-buffered formalin solution, demineralized in 5\% ethylenediaminetetraacetic acid, and embedded in paraffin. Paraffin sections were prepared using a slice thickness of $7 \mu \mathrm{m}$ and stained using hematoxylin and eosin (H-E staining).

\section{Results}

Body weight. Feeding activity of the experimental models (Figure 1) was normal, while no marked changes in weight were found for the duration of the experiment.

MR imaging of the mouse TMJ. The main components of the joint, temporal bone, joint disc and mandibular condyles were clearly depicted. The coronal section on T1W GE-MR image shows the temporal bone and mandibular condyle using low signal, and the articular joint disc using low-middle signal. Comparison of MR images at 3, 4, 6 and 12 weeks showed no marked changes in the position and shape of the joint disc, and no findings suggested inflammatory changes, such as joint effusion, with no clear differences evident between experimental and control groups. No articular cartilage was identified in the MR images (Figure 2).

Histological findings by $H-E$ staining of mouse TMJ. Irregularity was observed in the arrangement of chondrocytes at 3 weeks (Figures 3B and F). At 6 weeks, a cell-free area was formed in the cartilage (Figures $3 \mathrm{C}$ and $\mathrm{G}$ ), while cluster formation was observed at 12 weeks (Figures $3 \mathrm{D}$ and $\mathrm{H}$ ). No obvious changes in bone structure were observed (Figures 3A-H). 

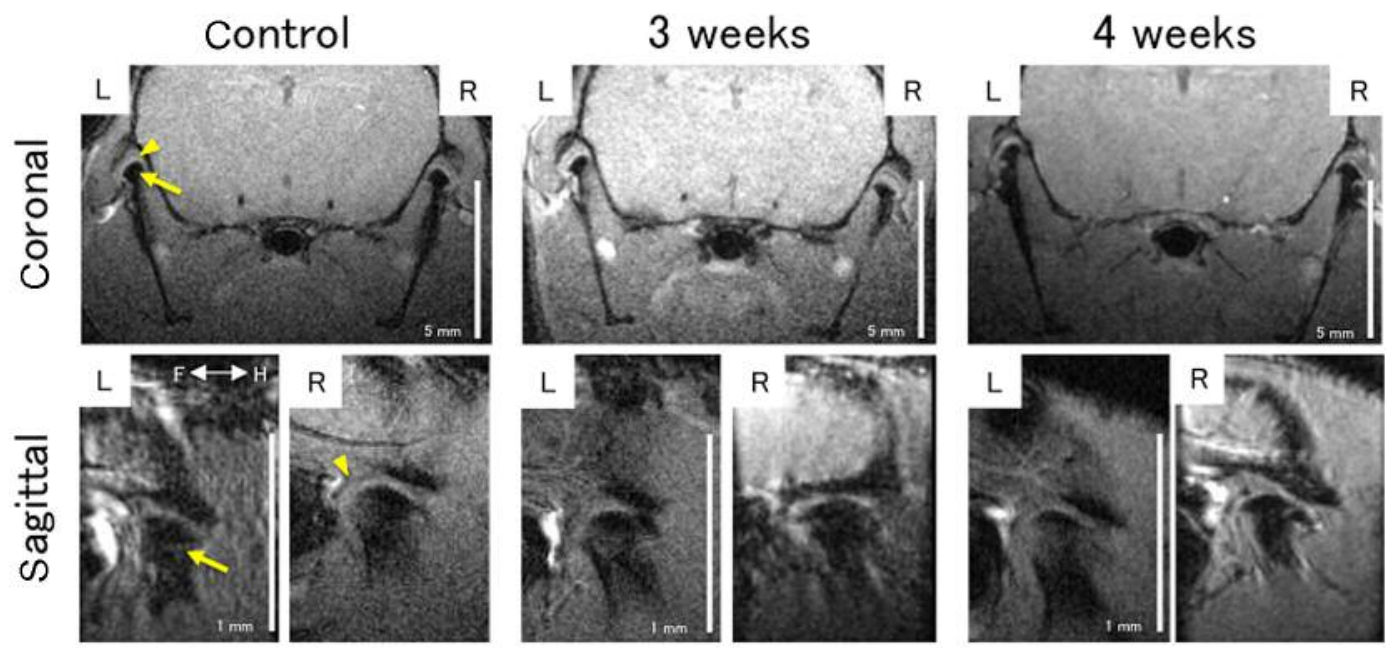

Control

6 weeks

12 weeks
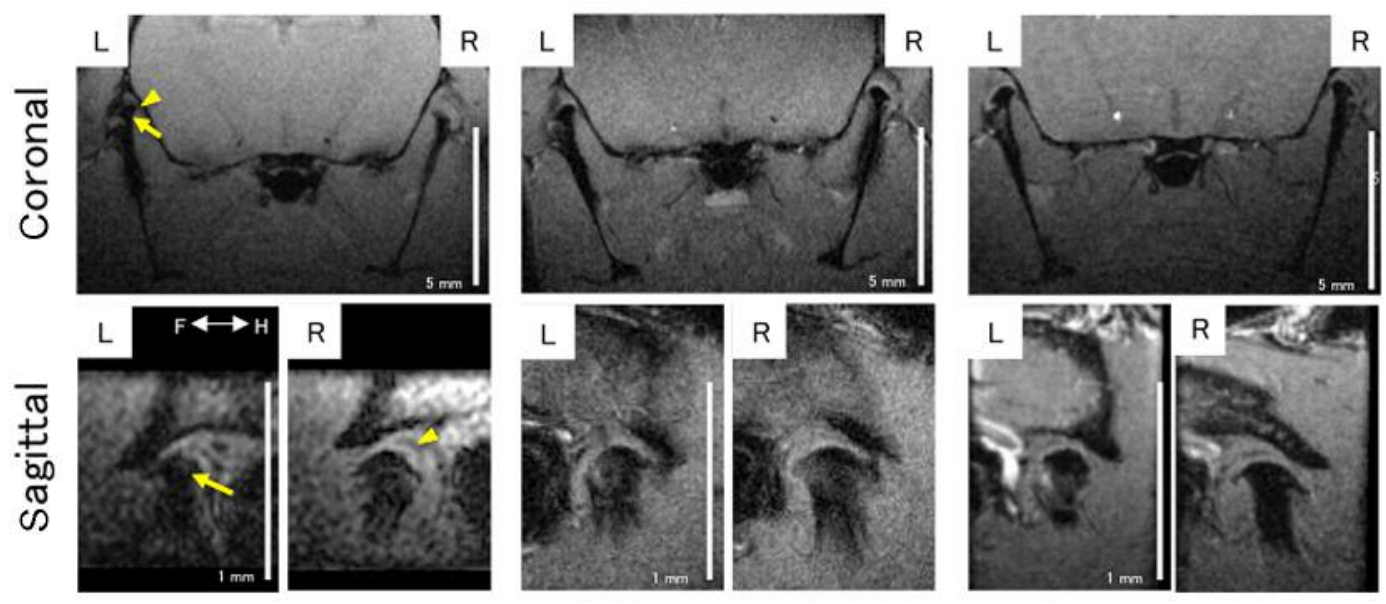

Figure 2. MR microimaging. T1-weighted GE-MR image shows the temporal bone and mandibular condyle (arrow) with low signal, and an articular joint disc (arrowhead) with low-middle signal. Comparison of MR images at 3, 4, 6 and 12 weeks shows no marked changes in the position or shape of the joint disc. There is no difference between the experimental and the control group.
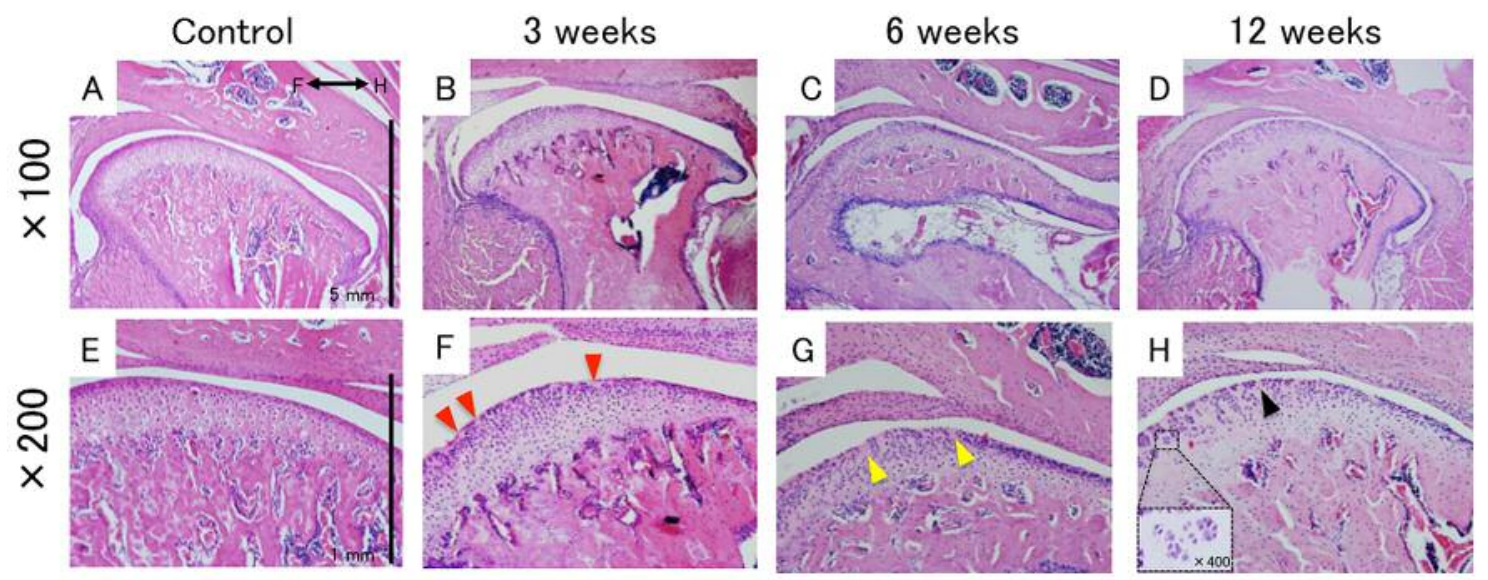

Figure 3. Histological findings of the mandibular condyle. (A-E) No obvious changes in bone structure are apparent. (F) Irregularity is observed in the arrangement of chondrocytes at 3 weeks (red arrowhead). $(G)$ At 6 weeks, a cell-free area is apparent in the cartilage (yellow arrowhead). (H) At 12 weeks, cluster formation is observed (black arrowhead). 


\section{Discussion}

In this study, we found that over time there was no change in the MR imaging appearance of the TMJ but there were histologic change. By maintaining an occlusion from anterior crossbite with metal tube, irregular cell arrangement, a cellfree area, and clustering were observed in the cartilage of the mandibular condyle. These histological changes were not detected through MR microimaging.

Imaging of living mice is also technically difficult. As the time required for MR imaging under general anesthesia increases, so does the burden on the experimental animal. The TMJ of the mouse is less than $3 \mathrm{~mm}$ in maximum dimension, including the joint disc and mandibular condyles, so high-field 7T MR microimaging is needed to observe such a small anatomical region. Few reports have looked into the TMJ in living experimental animals, and none has performed MR imaging several times on the same experimental animal. We placed a coil on the top of the head to examine bilateral TMJs in the same scan. The reason behind this was that in the experimental model we used a metal tube attached to the left incisor, disordering the occlusal load. Although no significant differences were seen between right and left MR imaging, the position of the articular disc and the structure of the temporal bone and mandible were clearly shown. The cartilage layer was not visible even with high-field 7T MR microimaging. Currently, in clinical practice, 3T MR imaging is used as high-power field equipment, however, detecting pathological changes on the level of the cartilage of the mandibular condyle is difficult, with the exception of large cartilage masses, such as in osteochondromatosis $(12,13)$.

Cluster formation was found in the cartilage layer on the mandibular condyle. Cluster formation in the cartilage is known to result from abnormal cellular activity (14). However, the cluster formation of chondrocytes shows higher cell division ability compared to normal chondrocytes, and it has also been suggested that cartilage may be repaired $(15,16)$. Hence, cluster formation in this experiment may be considered as a mechanosensitive process of adapting to a dynamic load.

In conclusion, we successfully performed MR imaging depicting the detailed structure of very small TMJs several times in the same living mice. This study can be applied for disease models of various etiologies, and should contribute to the elucidation of the pathophysiology of TMJ disease in the future, despite the fact that detecting changes at a cellular level in the cartilage layer of the mandibular condyle may still prove difficult.

\section{Conflicts of Interest}

The Authors declare no conflicts of interest associated with this manuscript.

\section{Authors' Contributions}

Conception and design of study were done by KI, FY and TK. Acquisition of data was done by KI, FY, SN and KT. Drafting the manuscript was done by KI, FY and SN, while revising the manuscript critically for important intellectual content was done by TK and AG.

\section{References}

1 Polat ME and Yanik S: Efficiency of arthrocentesis treatment for different temporomandibular joint disorders. Int $\mathrm{J}$ Oral Maxillofac Surg 17: S0901-5027(19)31303-7, 2019. PMID: 31540790. DOI: 10.1016/j.ijom.2019.08.017

2 Sperry MM, Kartha S, Winkelstein BA and Granquist EJ: Experimental methods to inform diagnostic approaches for painful TMJ. Osteoarthritis 98: 388-397, 2019. PMID: 30819041. DOI: $10.1177 / 0022034519828731$

3 Lee PP, Stanton AR, Schumacher AE, Truelove E and Hollender LG: Osteoarthritis of the temporomandibular joint and increase of the horizontal condylar angle: a longitudinal study. Oral Surg Oral Med Oral Pathol Oral Radiol 127: 339-350, 2019. PMID: 30709753. DOI: 10.1016/j.00oo.2018.12.014

4 Kyllar M, Putnová B, Jekl V, Stehlík L, Buchtová M and Štembírek J: Diagnostic imaging modalities and surgical anatomy of the temporomandibular joint in rabbits. Lab Anim 52: 38-50, 2018. PMID: 28443382. DOI: $10.1177 / 0023677$ 217702178

5 Yamazaki F, Satoh K and Seo Y: Structure and size-selective permeability of the synovial membrane of the temporomandibular joint of the mouse measured by MR imaging at 7T. Magn Reson Med Sci 14(2): 115-122, 2015. PMID: 25500776. DOI: $10.2463 / \mathrm{mrms} .2014-0058$

6 Embree MC, Iwaoka GM, Kong D, Martin BN, Patel RK, Lee AH, Nathan JM, Eisig SB, Safarov A, Koslovsky DA, Koch A, Romanov A and Mao JJ: Soft tissue ossification and condylar cartilage degeration following TMJ disc perforation in a rabbit pilot study. Osteoarthritis Cartilage 23(4): 629-639, 2015. PMID: 2557397. DOI: 10.1016/j.joca.2014.12.015

7 Wang XD, Kou XX, Mao JJ, Gan YH and Zhou YH: Sustained inflammation induces degeneration of the temporomandibular joint. J Dent Res 91(5): 499-505, 2012. PMID: 22427270. DOI: $10.1177 / 0022034512441946$

8 Long $X$ and Goss AN: A sheep model of intracapsular condylar fracture. J Oral Maxillofac Surg 65(6): 1102-1108, 2007. PMID: 17517292. DOI: 10.1016/j.joms.2006.06.307

9 Meng FW, Hu KJ, Kong L, Zhao YT, Liu YP and Zhou SX: Morphological evaluation of temporomandibular joint after open and closed treatment of type B diacapsular condylar fractures in sheep. Ann Anat 191(3): 288-293, 2009. PMID: 19303269. DOI: 10.1016/j.aanat.2008.12.002

10 Kaduk WM and Koppe T: Metric analysis of the upper space of the temporomandibular joint (TMJ) in pigs (Sus scrofadomestica) for evaluation of the pig as a model for arthroscopic TMJ surgery. Ann Anat 189(4): 367-370, 2007. PMID: 17695994. DOI: 10.1016/j.aanat.2007.02.015

11 Liu YD, Liao LF, Zhang HY, Lu L, Jiao K, Zhang J, He JJ, Wu YP, Chen D and Wang MQ: Reducing dietary loading decreases mouse temporomandibular joint degradation induced by anterior 
crossbite prosthesis. Osteoarthritis Cartilage 22(2): 302-312, 2014. PMID: 24316289. DOI: 10.1016/j.joca.2013.11.014

12 Manoliu A, Spinner G, Wyss M, Emi S, Nanz D, Ulbrich EJ, Gallo LM and Andreisek G: Quantitative and qualitative comparison of MR imaging of the temporomandibular joint at 1.5 and $3.0 \mathrm{~T}$ using an optimized high-resolution protocol. Dentomaxillofac Radiol 45(1): 20150240, 2016. PMID: 26371077. DOI: $10.1259 / \mathrm{dmfr} .20150240$

13 Sawada K, Amemiya T, Hirai S, Hayashi Y, Suzuki T, Honda M, Sisounthone $\mathrm{J}$, Matsumoto $\mathrm{K}$ and Honda $\mathrm{K}$ : Diagnostic reliability of 3.0-T MRI for detecting osseous abnormalities of the temporomandibular joint. J Oral Sci 60(1): 137-141, 2018. PMID: 29576573. DOI: 10.2334/josnusd.16-0838

14 Kubota Y, Takatsuka S, Nakagawa K and Yamamoto E: A model for temporomandibular joint disc repositioning surgery. J Oral Maxillofac Surg 59(12): 1443-1451, 2001. PMID: 11732032. DOI: $10.1053 /$ joms.2001.28279
15 Cledes G, Felizardo R, Foucart JM and Carpentier P: Validation of a chemical osteoarthritis model in rabbit temporomandibular joint: a compliment to biomechanical models. Int $\mathrm{J}$ Oral Maxillofac Surg 35(11): 1026-1033, 2006. PMID: 16829037. DOI: $10.1016 /$ j.ijom.2006.05.003

16 Duygu G, Güler N, Çam B and Kürkçü M: The effects of high molecular weight hyaluronic acid (Hylan G-F 20) on experimentally induced temporomandibular joint osteoartrosis: part II. Int J Oral Maxillofac Surg 40(12): 1406-1413, 2011. PMID: 21889876. DOI: 10.1016/j.ijom.2011.07.909

Received October 8, 2019

Revised October 21, 2019

Accepted October 22, 2019 